

\title{
Estimation of the Sensitive Volume for Gravitational-wave Source Populations Using Weighted Monte Carlo Integration
}

\author{
Vaibhav Tiwari \\ Cardiff School of Physics and Astronomy, Cardiff University, Queens Buildings, The \\ Parade, Cardiff CF24 3AA, UK \\ E-mail: tiwariv@cardiff.ac.uk
}

\begin{abstract}
The population analysis and estimation of merger rates of compact binaries is one of the important topics in gravitational wave astronomy. The primary ingredient in these analyses is the population-averaged sensitive volume. Typically, sensitive volume, of a given search to a given simulated source population, is estimated by drawing signals from the population model and adding them to the detector data as injections. Subsequently injections, which are simulated gravitational waveforms, are searched for by the search pipelines and their signal-to-noise ratio (SNR) is determined. Sensitive volume is estimated, by using Monte-Carlo (MC) integration, from the total number of injections added to the data, the number of injections that cross a chosen threshold on SNR and the astrophysical volume in which the injections are placed. So far, only fixed population models have been used in the estimation of binary black holes (BBH) merger rates. However, as the scope of population analysis broaden in terms of the methodologies and source properties considered, due to an increase in the number of observed gravitational wave (GW) signals, the procedure will need to be repeated multiple times at a large computational cost. In this letter we address the problem by performing a weighted MC integration. We show how a single set of generic injections can be weighted to estimate the sensitive volume for multiple population models; thereby greatly reducing the computational cost. The weights in this MC integral are the ratios of the output probabilities, determined by the population model and standard cosmology, and the injection probability, determined by the distribution function of the generic injections. Unlike analytical/semi-analytical methods, which usually estimate sensitive volume using single detector sensitivity, the method is accurate within statistical errors, comes at no added cost and requires minimal computational resources.
\end{abstract}

PACS numbers: 04.30.-w, 04.80.Nn

Keywords: Binary black hole merger rate, Sensitive volume 


\section{Introduction}

Understanding source population or a mixture of them is one of the primary goal of gravitational wave astronomy. These studies include population analysis, estimating rate of occurrence of an astrophysical phenomenon or placing upper-limit on the occurrence of a proposed astrophysical phenomenon in the event of a null observation. Prior to the observation of GW150914 [1] LIGO published upper limits on the merger rate of stellar mass compact binaries after all the scientific runs. These include upper limits, as a function of total mass for BBH and neutron star-black hole (NSBH) binaries [2], for binaries with first mass fixed at $1.35 \mathrm{M}_{\odot}$ and second mass uniformly distributed between $2 \mathrm{M}_{\odot}$ and $20 \mathrm{M}_{\odot}$ [3], for fixed mass binary neutron stars (BNS), BBH and NSBH binaries [4], and for BBH on the component mass plane [5, 6]. The observation of GW150914 during LIGO's first observation run and the observation of GW170817 during the second observation run provided opportunity for the first time for the estimation of the $\mathrm{BBH}$ and the BNS merger rate [7, 8]. For example, the rate upper limit on BNS mergers, estimated at the end of LIGO's sixth scientific run, was $\sim 10^{-4} \mathrm{Mpc}^{-3} \mathrm{yr}^{-1}$ at 90\% confidence, while the BNS merger rate estimated after the observation of GW170817 is $\sim 10^{-6} \mathrm{Mpc}^{-3} \mathrm{yr}^{-1}$ [6, 8].

Sources such as intermediate mass binary black holes (IMBH) and NSBH are anticipated for observation and as of yet only upper limits on the merger rate have been published [9, 10]. Estimated rate limit, for IMBH sources, at the end of LIGO's first observation run, is $0.93 \mathrm{Gpc}^{-3} \mathrm{yr}^{-1}$, while for NSBH binaries it is $3600 \mathrm{Gpc}^{-3} \mathrm{yr}^{-1}$ [11, 12, 13]. Rate limit on eccentric binaries have also been estimated [14].

The primary ingredient in the estimation of merger rate or upper-limit on the merger rate [15] is the sensitive volume. In the event of a non-detection, upper rate limits have been placed for source populations. Under the assumption that observation of a GW signal is a Poisson process, the rate limit, quoted for a chosen confidence interval, is inversely proportional to the sensitive volume of the source population. For a source population of compact binaries, which has resulted in an observation, if the intrinsic, redshift independent, rate of coalescence is $R$, then one can expect to observe $R\langle V T\rangle$ number of GW signals. But, as the intrinsic rate is not known the variables are reversed to estimate the expected rate [7, 16],

$$
R=\frac{N_{\text {obs }}}{\langle V T\rangle},
$$

where $\langle V T\rangle$ is the time-volume product, computed using the population averaged sensitive volume $\langle V\rangle$ and time $T$ during which $N_{\text {obs }}$ observations have been made. One can perform a more sophisticated rate estimate e.g. while including the confidence with which observations have been made [17], but the primary ingredient is the sensitive volume.

Observation of the first GW signal also opened the window for population analysis. The first result estimated the probability distribution of the exponent under the assumption that mass distribution of the black holes follows a power-law distribution 
[16]. Since then additional work has gone into understanding the spin distribution of the black holes [18, 19]. With the increase in the number of observations, it is expected, that the scope of population analysis will broaden in terms of methodologies and source properties considered. Sensitive volume is a primary ingredient in the Bayesian framework of population analysis as it accounts for the selection effects [20, 16]. A range of sensitive volumes, corresponding to different population models, are required. Large scale simulations for the estimation of sensitive volume is computationally expensive and performing multiple analyses dedicated at the source populations is not computationally viable.

\section{Estimation of sensitive volume of a search}

The population averaged time-volume is defined as,

$$
\langle V T\rangle=\int \mathrm{d} z \mathrm{~d} \theta \frac{\mathrm{d} V_{c}}{\mathrm{~d} z} \frac{1}{1+z} p_{\text {pop }}(\theta) f(z, \theta) \cdot T,
$$

where $\mathrm{d} V_{c} / \mathrm{d} z$ is the differential co-moving volume, $p_{\text {pop }}(\theta)$ is the distribution function for the astrophysical population and $f(z, \theta)$ is the probability of recovering a signal, with parameters $\theta$, at a redshift $z$. The additional factor of $1+z$ in the denominator accounts for the time-dilation in the intrinsic rate caused by the expanding universe [7]. Equation 2 is estimated by using Monte-Carlo integration [25]. Signals are drawn from the population model and added to the detector data as injections. The injections are placed in redshift as determined by the standard cosmology. The probability of making an injection at redshift $z$ is given by,

$$
p(z)=\frac{\mathrm{d} V_{c} / \mathrm{d} z}{(1+z) V_{0}}
$$

where $V_{0}$ is the astrophysical volume in which injections have been made corresponding to a maximum redshift $z_{\max }$,

$$
V_{0}=\int_{0}^{z_{\max }} \frac{\mathrm{d} V_{c}}{\mathrm{~d} z} \frac{1}{1+z} \mathrm{~d} z
$$

The injections are searched for by search pipelines and their SNR is determined [21, 22, 23, 24]. An injection is categorized as recovered if it crosses a chosen SNR

threshold. Sensitive volume, in terms of the number of injected signals $N_{\text {inj }}$ and the number of recovered signals $N_{\text {rec }}$, is given by [7, 16],

$$
\langle V\rangle=V_{0} \frac{N_{\text {rec }}}{N_{\text {inj }}} .
$$

Such an analysis suffers from two drawbacks: (i) The distribution function $s(\theta)$ is fixed, hence, multiple injection runs will be required if sensitive volume is needed for a range of population models, and (ii) as the redshift placement of the injections is independent of the parameters $\theta$, significant number of injections don't contribute, due to being placed at redshift values where their recovery is impossible. 
In the following section we overcome these limitations by performing a weighted $\mathrm{MC}$ integration on a set of generic injections. In Section 3 we describe the analysis and present results and conclusion in Section 4.

\section{Analysis}

The shortcomings listed in the previous section can be overcome by using generic injection sets followed by weighting the injections in the MC integration. The applied weight is the ratio of the output probability, determined by the population model and standard cosmology, and the injection probability, determined by the distribution function of the generic injections. This allows the use of the same set of injections in the estimation of sensitive volume for different population models. The weight $w$ on the $i^{\text {th }}$ injection reads,

$$
w_{i}=\frac{p_{\text {pop }}\left(\left\{m_{1}, m_{2}, \vec{s}_{1}, \vec{s}_{2}\right\}_{i}\right) \cdot p\left(z\left(d_{i}\right)\right)}{p_{\text {inj }}\left(\left\{m_{1}^{d}, m_{2}^{d}, \vec{s}_{1}, \vec{s}_{2}, d\right\}_{i}\right) \cdot J_{i}} .
$$

The output probability, in the numerator, depends on the probability distribution of the target population model $p_{\text {pop }}$, defined in terms of the source frame masses $\left(m_{1}\right.$, $\left.m_{2}\right)$ and the dimensionless spins $\left(\vec{s}_{1,2} \equiv\left\{s_{x}, s_{y}, s_{z}\right\}_{1,2}\right)$, and the redshift probability, determined by the standard cosmology and as defined in Equation 3. The maximum redshift, $z_{\max }$, defines the boundary beyond which events produced by the population model are not recoverable by the detector network. The input probability $p_{\text {inj }}$, in the denominator, depends on the injection distribution and is defined in terms of the detector frame masses $\left(m_{1}^{d}, m_{2}^{d}\right)$, spins $\left(\vec{s}_{1,2}\right)$ and luminosity distance $d$. The Jacobian, $J$, maps the probability from the detector frame parameters, $\left(m_{1}^{d}, m_{2}^{d}, d\right)$, to the source frame parameters $\left(m_{1}, m_{2}, z\right)$,

$$
J_{i}=\left(1+z\left(d_{i}\right)\right)^{2}\left(\frac{\partial d}{\partial z}\right)_{i} .
$$

Due to red-shifting, the signal produced from a binary with masses $m_{1}$ and $m_{2}$ is observed in the detector frame with masses $m_{1}^{d}=(1+z) m_{1}$ and $m_{2}^{d}=(1+z) m_{2}$. Moreover, if a binary merges at a redshift $z$, the merger is observed, by the detector, at the corresponding luminosity distance. The amplitude of the signal, as observed by the detector, is inversely proportional to the luminosity distance. Hence, it is convenient to perform injections using detector based quantities.

Injections should adequately cover the parameter space i.e. sufficient number of injections should be made, in parts of the parameter space, where $p_{\text {pop }}$ is non-negligible. Moreover, redshift placement should ensure that injections are placed wherever the recovery probability, $f(z, \theta)$, is non-zero. It is better to have a quantitative measure reflecting the goodness of coverage, but, however, for the population models that are only dependent on the component masses a good assessment can be based on how well injections cover the population model on the source frame component mass-plane. 
Adequate coverage in redshift can be ascertained by injecting distance uniform in chirp distance with a large enough fiducial distance.

This analysis does not need dedicated injection runs. Injection runs are regularly performed to assess efficiency of the search pipelines in different regions of the parameter space [21, 22, 23, 24]. Table 1] summarizes the most commonly used parameter distributions used in making injections. Usually, multiple injection sets, covering different regions of masses and spins, are used to adequately cover the parameter space and are ideal for the use of the presented analysis (if the same number of injections are performed from $k$ injections set, the input probability is summed over all the injection distributions in the set, i.e. $p_{\text {inj }}\left(m_{1}^{d}, m_{2}^{d}, s_{1 z}, s_{2 z}, d\right)=\sum_{j=1}^{k} p_{\text {inj }}^{j}\left(m_{1}^{d}, m_{2}^{d}, s_{1 z}, s_{2 z}, d\right)$, $p_{\text {inj }}^{j}(\cdots)=p^{j}\left(m_{1}^{d}, m_{2}^{d}\right) \cdot p^{j}\left(s_{1 z}\right) \cdot p^{j}\left(s_{2 z}\right) \cdot p^{j}(d)$ being the injection distributions for the $j^{t h}$ injection set).

Weighting maps the injections to the population model and cosmological redshift distribution, but, astrophysical volume in which injections are made can not be estimated using Equation 4. Input probability can also be expressed in terms of the parameters $\left(\mathcal{M}^{d}, q, \overrightarrow{s_{1}}, \vec{s}_{s}\right)$, where $q=m_{2} / m_{1}$ is the mass ratio. Out of these parameters, only parameter that changes value from source to detector frame is the chirp mass. Unlike the injections sampled from the population model, that cut a rectangular shape, the generic injections are characterised by the detector frame quantities and cut an irregular shape in the $\mathcal{M}-z$ plane. Redshift placement of the injections depend on the source frame chirp mass. The astrophysical volume in which injections are made is given by,

$$
V_{0}^{m}=\int_{\mathcal{M}_{\min }}^{\mathcal{M}_{\max }} \int_{z_{\min }(\mathcal{M})}^{z_{\max }(\mathcal{M})} p_{\text {pop }}(\mathcal{M}) \frac{\mathrm{d} V_{c}}{\mathrm{~d} z} \frac{1}{1+z} \mathrm{~d} z \mathrm{~d} \mathcal{M},
$$

where $p_{\text {pop }}(\mathcal{M})$ is the population model expressed as a function of source frame chirp mass after being marginalized over other parameters. The boundaries $z_{\max }(\mathcal{M})$ and $z_{\text {min }}(\mathcal{M})$ are determined by the injection sets, while the boundaries $\mathcal{M}_{\text {min }}$ and $\mathcal{M}_{\max }$ are set by the population model. Estimation of Equation $V_{0}^{m}$ is not straightforward and we resort to $\mathrm{MC}$ integration again. We simulate a physical source population by sampling chirp masses from the population model and redshifts according to distribution in Equation 3. We calculate the detector frame chirp mass and luminosity distances of these samples and count the number of samples that have non-zero injection probability in the $\mathcal{M}^{d}-d$ space ( $p_{\text {inj }}$ expressed as a function of $\mathcal{M}^{d}$ and $d$ and marginalized over other parameters). The MC estimate of $V_{0}^{m}$ is given by,

$$
V_{0}^{m}=V_{0} \frac{N_{\text {inside }}}{N_{\text {samples }}},
$$

where $N_{\text {inside }}$ is the total number of samples that have non-zero injection probability and $N_{\text {samples }}$ is the total number of samples drawn from the population model. $V_{0}$ is given by Equation 4 corresponding to the maximum redshift $z_{\max }$. If the computational cost of sampling chirp mass is low, one can generate a large number of samples to safely ignore statistical errors incurred in this MC integration. Finally, sensitive volume is estimated 


\begin{tabular}{cc}
\hline Distribution uniform in & Probability Density \\
\hline Component Mass & $p\left(m_{1}^{d}, m_{2}^{d}\right)=\frac{1}{\left(m_{\max }^{d}-m_{\min }^{d}\right)^{2}}$ \\
Total Mass & $p\left(m_{1}^{d}, m_{2}^{d}\right)=\frac{1}{\left(m_{\max }^{d}-m_{\min }^{d}\right)\left(m_{1}^{d}+m_{2}^{d}-2 m_{\min }^{d}\right)}$ \\
Aligned Spin & $p\left(s_{z}\right)=\frac{1}{s_{z, \max }-s_{z, \min }}$ \\
Total Spin & $p\left(s_{z}\right)=\frac{\log \left(s_{\max }-\log \left(\left|s_{z}\right|\right)\right)}{2 s_{\max }}$ \\
Distance & $p(d)=\frac{1}{d_{\max }-d_{\min }}$ \\
Chirp Distance & $p(d)=\frac{\mathcal{M}_{\mathrm{BNS}}^{(5 / 6)}}{\left(\mathcal{M}^{d}\right)^{(5 / 6)}\left(d_{\max }-d_{\min }\right)}$ \\
\hline
\end{tabular}

Table 1: The probability density of some of the mass, spin and distance distribution used in performing injections. Typically an injection run will select a distribution for mass, spin and distance, each. For the first distribution, in this table, both the component mases are uniformly distributed between the minimum value, $m_{\min }^{d}$, and the maximum value, $m_{\max }^{d}$. For the second distribution, both the component masses have the same minimum and the maximum value. $m_{1}^{d}$ is uniformly distributed between $m_{\text {min }}^{d}$ and $M^{d}-m_{\min }^{d}$. The total mass, $M^{d}=m_{1}^{d}+m_{2}^{d}$, is uniformly distributed between $M_{\min }^{d}=2 m_{\min }^{d}$ and $M_{\max }^{d}=m_{\min }^{d}+m_{\max }^{d}$. For the third distribution, pertaining to spins, $s_{z}$ is uniformly distributed between $s_{z \text {,min }}$ and $s_{z \text {,max }}$; other spin components are zero. For the fourth distribution, spin magnitudes are uniformly distributed between $s_{\min }$ and $s_{\max }$; other spin components follow the same distribution with $s_{z}$ replaced by $s_{x}$ or $s_{y}$. For the fifth distribution, pertaining to luminosity distance, $d$ is uniformly distributed between $d_{\min }$ and $d_{\max }$. For the sixth distribution, $d$ is based on the mass distribution and is uniformly distributed between $d_{\min }\left(\mathcal{M}^{d}\right)^{(5 / 6)}$ and $d_{\max }\left(\mathcal{M}^{d}\right)^{(5 / 6)}$, where $d_{\min }$ and $d_{\min }$ are fixed minimum and maximum fiducial distances, (called as chirp distance in technical publications). $\mathcal{M}_{\mathrm{BNS}}$ is the chirp mass of a $1.4 \mathrm{M}_{\odot}-1.4 \mathrm{M}_{\odot} \mathrm{BNS}_{\text {, }}$ where chirp mass is defined as, $\mathcal{M}^{d}=\left(m_{1}^{d} m_{2}^{d}\right)^{(3 / 5)} /\left(m_{1}^{d}+m_{2}^{d}\right)^{(1 / 5)}$. This distribution exploits the approximate dependence of the detectability of a binary on its chirp mass to avoid placement of significant number of injections at redshifts where their recovery is impossible (the maximum distance at which an injection is recovered at a high enough SNR to be detectable is roughly dependent on the chirp mass of the binary).

by putting Equation 6 and Equation 9 together,

$$
\langle V\rangle=V_{0}^{m} \frac{\sum_{i \in \operatorname{Rec}} w_{i}}{\sum_{i \in \operatorname{Inj}} w_{i}} .
$$

The MC integration has errors associated with it. The error, in the mean weight, 
in the denominator of Equation 10 is given by,

$$
\left(N_{\text {inj }} \delta w\right)^{2}=\frac{N_{\text {inj }} \sum_{i \in \text { inj }} w_{i}^{2}-\left(\sum_{i \in \text { inj }} w_{i}\right)^{2}}{N_{\text {inj }}} .
$$

The same expression holds true for the numerator but summed over recovered events (the weight for a missed injection is zero). Propagating the errors to find the error in the ratio gives

$$
\frac{\delta\langle V\rangle}{\langle V\rangle}=\sqrt{\sum_{k=\text { inj,rec }} \frac{N_{\text {inj }} \sum_{i \in k} w_{i}^{2}-\left(\sum_{i \in k} w_{i}\right)^{2}}{N_{\text {inj }}\left(\sum_{i \in k} w_{i}\right)^{2}}} .
$$

$N_{\text {inj }}$ is of the order of few thousands and the number of the recovered events is only a small fraction of the injected events, additionally, irrespective of the value of the weights, the sum of squares of the weights is much less than the square of the sum of the weights. Under these conditions the dominant term is $\sum_{\operatorname{Rec}} w_{i}^{2} /\left(\sum_{\operatorname{Rec}} w_{i}\right)^{2}$ (for example, with a total 100000 injections and ten percent recovered injections, this term is around ten times the remaining terms). The error in the sensitive volume reduces to,

$$
\frac{\delta\langle V\rangle}{\langle V\rangle}=\frac{\sqrt{\sum_{\operatorname{Rec}} w_{i}^{2}}}{\sum_{\operatorname{Rec}} w_{i}} .
$$

In Section 4 we apply the algorithm and obtain some results.

\section{Results and Conclusion}

In this section we estimate the time-volume product for the same stretch of data that was used in estimating an updated BBH merger rate after the GW observation GW170104 [26]. The results discussed in this section are based on the injection runs performed using the search pipeline PyCBC [21, 22].

So far the two population models that have been used in the estimation of $\mathrm{BBH}$ merger rate are as follows [16]:

a Uniform in the logarithm of the component masses, with combined probability density given by $p\left(m_{1}, m_{2}\right) \propto m_{1}{ }^{-1} m_{2}{ }^{-1}$ and

b The primary mass follows a power-law distribution while the secondary mass is uniformly distributed between smallest mass and the primary mass, $p\left(m_{1}\right) \propto m_{1}^{-2.35}$ with a uniform distribution on the second mass.

The masses are required to be $5 \mathrm{M}_{\odot} \leq m_{2} \leq m_{1}$ and $m_{1}+m_{2} \leq 100 \mathrm{M}_{\odot}$. Spins have been chosen to be aligned and uniformly distributed between -0.99 and 0.99. Sensitive volume 


\begin{tabular}{ccc}
\hline $\begin{array}{c}\text { Population } \\
\text { Model }\end{array}$ & $\begin{array}{c}100\langle V T\rangle \\
\text { direct injections }\end{array}$ & $\begin{array}{c}100\langle V T\rangle \\
\text { scaled injections }\end{array}$ \\
\hline Uniform in log & $4.61 \pm 0.1$ & $4.54 \pm 0.13$ \\
\hline Power-law & $1.38 \pm 0.03$ & $1.36 \pm 0.06$ \\
\hline
\end{tabular}

Table 2: Comparison of time volume product, in units of $\mathrm{Gpc}^{3}-\mathrm{yr}$, estimated by using injections runs drawn from population models and by scaling injections in a generic injection set. The result correspond to calculation of rates corresponding to the event GW170104 [26].

has been estimated by performing MC integral using injections with parameters directly sampled from the population models. The statistical error in the MC integration for this analysis is $\delta\langle V\rangle=\sqrt{\epsilon(1-\epsilon) / N_{\text {Inj }}}$, where $\epsilon$ is the efficiency of recovering injections.

We also estimate the sensitive volume, using the weighted $\mathrm{MC}$ integration. We use six different injection distributions. For all the distributions, the injections probability of both the component masses is uniform over the logarithm of the component masses $\left(p\left(m_{1}\right) \propto m^{-1}\right)$, with component mass range:

a Component mass range: $4 \mathrm{M}_{\odot}$ to $180 \mathrm{M}_{\odot}$,

b Component mass range: $6 \mathrm{M}_{\odot}$ to $180 \mathrm{M}_{\odot}$,

c Component mass range: $10 \mathrm{M}_{\odot}$ to $180 \mathrm{M}_{\odot}$,

d Component mass range: $16 \mathrm{M}_{\odot}$ to $180 \mathrm{M}_{\odot}$,

e Component mass range: $24 \mathrm{M}_{\odot}$ to $180 \mathrm{M}_{\odot}$,

f Component mass range: $36 \mathrm{M}_{\odot}$ to $180 \mathrm{M}_{\odot}$.

Spin distribution is aligned and uniformly distributed between -0.99 and 0.99 , hence, the weights in the MC integral depends only on the component masses.

Figure 1 shows that the injection sets adequately cover the two population models on the component mass plane. Figure also plots the weights on the MC integration for the recovered injections. The injections follow a uniform distribution in chirp distance with maximum fiducial distance set to $300 \mathrm{Mpc}$. Current sensitivity of the detector network is less than $200 \mathrm{Mpc}$ for $1.4-1.4 \mathrm{M}_{\odot}$ BNS. The maximum distance scales to around 4 Gpc for a GW150914 like binary. This maximum distance is much larger than the distances to which GW150914 like binary can be observed. Similar calculation for other GW observation suggests that the maximum luminosity distance to which injections are placed is much larger than the current reach for the detectors. The affect of mass ratios and spins on this assessment is negligible. Table 2 compares the time volume product for the two models obtained using both the methods. Although the mean values are close, the statistical errors are larger for the case of weighted MC integration. Increasing the number of injections has a direct impact on the error. The error will reduce approximately as $1 / \sqrt{F}$, where $F$ is the factor by which number of injections is increased. In fact, we expect when analyzed over the whole data obtained during LIGO's second observation run, statistical errors will reduce to around $1 \%$. 

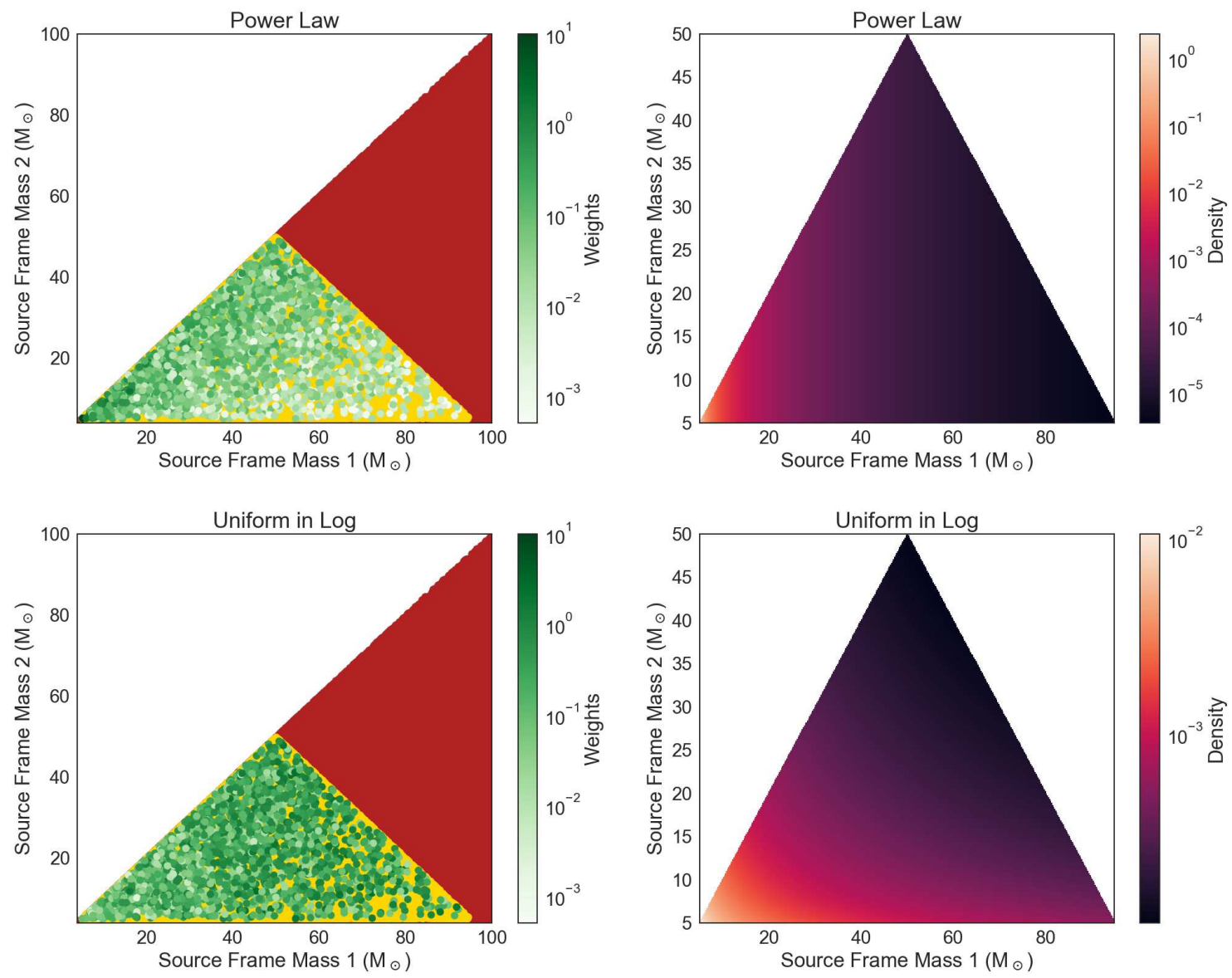

Figure 1: The red dots in the figure plot the source frame masses of all the injections. Yellow dots are the source frame masses for the injections which have a non-zero weight. The green dots are masses of the recovered injections which have a color depth coding based on the value of the weights. In the plots the heavier of the two black holes is plotted as the first component mass. The maximum injected component mass is 180 $\mathrm{M}_{\odot}$, but for the sake of clarity, the axes have been truncated. Additionally, the weights for the yellow dots are not shown. The right hand side of the figure plots the probability density of the two population models. Injections adequately cover the population models on the source frame component mass plane.

The results obtained are promising and offer a way to estimate sensitive volume without enormous investment of user and computational time. The method is open to include a larger parameter space (precession, eccentricity, tides etc.) or phenomena like redshift dependence of star formation rates, etc. Sensitive volume is an important ingredient when performing population inference and the estimation of corresponding merger rates. Analytical models can be used to estimate sensitive volume but they are usually not accurate. Strength of the method lies in its fast and accurate calculation of sensitive volume every time parameters defining a population model are changed (for example see [28] and supplement material for [26]). 


\section{Acknowledgement}

The author would like to thank Gregory Mendel for providing very useful feedback on the manuscript, to Tom Dent for discussion on the injection distributions, to Albrecht Rudiger for multiple editorial fixes and to Stephen Fairhurst for multiple useful discussions on the topic. This work was supported by the STFC grant ST/1990s/1.

The authors thank to the LIGO Scientific Collaboration for access to the data and gratefully acknowledge the support of the United States National Science Foundation (NSF) for the construction and operation of the LIGO Laboratory and Advanced LIGO as well as the Science and Technology Facilities Council (STFC) of the United Kingdom, and the Max-Planck-Society (MPS) for support of the construction of Advanced LIGO. Additional support for Advanced LIGO was provided by the Australian Research Council.

\section{References}

[1] B. Abbott et al. (LIGO Scientific Collaboration and Virgo Collaboration), Observation of Gravitational Waves from a Binary Black Hole Merger, Phys. Rev. Lett., Volume 116, 061102 (2016).

[2] B. Abbott et al. (LIGO Scientific Collaboration), Search of S3 LIGO data for gravitational wave signals from spinning black hole and neutron star binary inspirals, Phys. Rev. D, Volume 78, $042002(2008)$.

[3] B. Abbott et al. (LIGO Scientific Collaboration), Search for gravitational waves from binary inspirals in S3 and S4 LIGO data., Phys. Rev. D, volume 77, 062002 (2008).

[4] J. Abadie et al. (LIGO Scientific Collaboration, Virgo Collaboration), Search for gravitational waves from compact binary coalescence in LIGO and Virgo data from S5 and VSR1., Phys. Rev. D, Volume 82, 102001 (2010).

[5] J. Abadie et al. (LIGO Scientific Collaboration, Virgo Collaboration), Search for gravitational waves from binary black hole inspiral, merger, and ringdown., Phys. Rev. D, Volume 83, 122005 (2011).

[6] J. Abadie et al. (LIGO Scientific Collaboration, Virgo Collaboration), Search for gravitational waves from low mass compact binary coalescence in LIGOs sixth science run and Virgos science runs 2 and 3., Phys. Rev. D, Volume 85, 082002 (2012).

[7] Abbott et al. (LIGO Scientific Collaboration and Virgo Collaboration), The Rate of Binary Black Hole Mergers Inferred from Advanced LIGO Observations Surrounding GW150914, Astrophys. J. Lett., Volume 833, Number 1 (2016).

[8] Abbott et al. (LIGO Scientific Collaboration and Virgo Collaboration), GW170817: Observation of Gravitational Waves from a Binary Neutron Star Inspiral, Phys. Rev. Lett., Volume 119, 161101 (2017).

[9] J. Abadie et al. (LIGO Scientific Collaboration, Virgo Collaboration), Search for gravitational waves from intermediate mass binary black holes, Phys. Rev. D, Volume 85, 102004 (2012).

[10] J. Aasi et al. (LIGO Scientific Collaboration and Virgo Collaboration), Search for gravitational radiation from intermediate mass black hole binaries in data from the second LIGO-Virgo joint science run., Phys. Rev. D, Volume 89, 122003 (2014).

[11] Abbott et al. (LIGO Scientific Collaboration and Virgo Collaboration), Search for intermediate mass black hole binaries in the first observing run of Advanced LIGO, Phys. Rev. D, Volume 96, 022001 (2017).

[12] J. C. Bustillo, Sensitivity of gravitational wave searches to the full signal of intermediate-mass 
black hole binaries during the first observing run of Advanced LIGO, Phys. Rev. D, Volume 97, 024016 (2018).

[13] Abbott et al. (LIGO Scientific Collaboration and Virgo Collaboration), Upper limits on the rates of binary neutron star and neutron-star-black-hole mergers from Advanced LIGO's first observing run The Astro. J. Lett., Volume 832, Number 2 (2016).

[14] V. Tiwari et al. Proposed search for the detection of gravitational waves from eccentric binary black holes, Phys. Rev. D, Volume 93, 043007 (2016).

[15] R. Biswas et al. The loudest event statistic: general formulation, properties and applications, Class. and Quantum Grav., Volume 26, Number 17 (2009).

[16] Abbott et al. (LIGO Scientific Collaboration and Virgo Collaboration), Binary Black Hole Mergers in the first Advanced LIGO Observing Run, Phys. Rev. X, Volume 6, 041015 (2016).

[17] W. M. Farr et al., Counting and confusion: Bayesian rate estimation with multiple populations Phys. Rev. D, Volume 91, Number 023005 (2015).

[18] W. M. Farr et al., Distinguishing spin-aligned and isotropic black hole populations with gravitational waves, Nature, Volume 548, pages 426-429 (2017)

[19] Ben Farr, Will Farr and Daniel Holz, Using Spin to Understand the Formation of LIGO and Virgo's Black Holes, The Astro. J. Lett., Volume 854, Number 1 (2018).

[20] I. Mandel, W. M. Farr, and J. Gair, Technical Report No. P1600187, url:https://dcc.ligo.org/LIGOP1600187/public.

[21] S. A. Usman et al., The PyCBC search for gravitational waves from compact binary coalescence, Class. Quantum Grav., Volume 33, Number 21 (2016).

[22] T. D. Canton et al. Implementing a search for aligned-spin neutron star-black hole systems with advanced ground based gravitational wave detectors, Phys.Rev. D, Volume 90, 082004 (2014).

[23] Cody Messick et al., Analysis framework for the prompt discovery of compact binary mergers in gravitational-wave data, Phys. Rev. D, Volume 95, 042001 (2017).

[24] S. Klimenko et al., Method for detection and reconstruction of gravitational wave transients with networks of advanced detectors, Phys. Rev. D, Volume 93, 042004 (2016).

[25] M. E. J. Newman and G. T. Barkema, Monte Carlo Methods in Statistical Physics, chapter 1-4, Oxford University Press, 1999.

[26] Abbott et al. (LIGO Scientific Collaboration and Virgo Collaboration), GW170104: Observation of a 50-Solar-Mass Binary Black Hole Coalescence at Redshift 0.2, Phys. Rev. Lett., Volume 118,221101 (2017).

[27] Abbott et al. (LIGO Scientific Collaboration and Virgo Collaboration), Prospects for Observing and Localizing Gravitational-Wave Transients with Advanced LIGO and Advanced Virgo, Living Rev Relativ (2016) 19: 1. https://doi.org/10.1007/lrr-2016-1.

[28] Abbott et al. (LIGO Scientific Collaboration and Virgo Collaboration), Astrophysical Implications of the Binary Black-hole Merger GW150914, The Astro. J. Lett., Volume 818, Number 2 (2016). 